\title{
Analysis of murine and human Treg subsets in inflammatory bowel disease
}

\author{
XIAOYUN SUN ${ }^{1,2}$, SHAOHENG HE ${ }^{2}$, CHANGLONG LV ${ }^{1}$, XUN SUN $^{1}$, \\ JUNLING WANG $^{2}$, WENJIAO ZHENG ${ }^{2}$ and DANAN WANG ${ }^{1}$ \\ ${ }^{1}$ Department of Immunology, School of Basic Medical Science, China Medical University, Shenyang, Liaoning 110001; \\ ${ }^{2}$ Allergy and Clinical Immunology Research Centre, The First Affiliated Hospital of Jinzhou Medical University, \\ Jinzhou, Liaoning 121001, P.R. China
}

Received July 9, 2016; Accepted April 25, 2017

DOI: $10.3892 / \mathrm{mmr} .2017 .6912$

\begin{abstract}
Previous studies have indicated that regulatory $\mathrm{T}$ cells serve essential roles in maintaining intestinal homeostasis, however, the role of different Treg subsets in modulating inflammatory bowel disease has still not been addressed clearly. In the present study, the authors measured the percentage of Foxp $3^{+} \mathrm{IL}-10^{+} \mathrm{TGF}-\beta^{+}$natural Tregs, Foxp3IL- $10^{+}$TGF- $\beta{ }^{-}$induced Tregs, CD127- induced Tregs and CD8 ${ }^{+}$ Tregs at different time points in DSS-induced experimental colitis model in murine lamina propria lymphocytes, mesenteric lymph node and peripheral blood. In addition, the authors compared the frequency of four Treg subsets in patients diagnosed of ulcerative colitis at different stages with enrolled healthy controls. The percentage of Foxp $3^{+} \mathrm{IL}-10^{+}$ TGF- $\beta^{+}$natural Tregs decreased in acute stage of both human and mice was observed, but proliferated significantly during remittent stage. Foxp $3^{-}$IL- $10^{+}$TGF- $\beta^{-}$inducible (i) Treg and CD127-iTreg was observed as being significantly decreased percentage in LPL at 4 and 7 days, the frequency of Foxp3 IL- $10^{+}$TGF- $\beta$-iTreg cells became decreased and CD127-iTreg only slightly increased at the chronic stage following DSS induction. However, the proportion of both Foxp3- IL-10 ${ }^{+}$ TGF- $\beta^{-}$iTreg and CD127-iTreg was nearly unchanged in human IBD. Although intestinal inflammation decreased the percentage of $\mathrm{CD}^{+}$Tregs, it remained lower in the remittent stage of human IBD. Only enhanced proliferation of lamina propria lymphocytes-derived $\mathrm{CD}^{+}$Treg was reported at 7 days in dextran sodium sulfate-induced murine colitis. The results demonstrated that Foxp $3^{+} \mathrm{IL}-10^{+} \mathrm{TGF}-\beta^{+}$natural Tregs may serve an essential role in exhibiting suppressive
\end{abstract}

Correspondence to: Professor Danan Wang, Department of Immunology, School of Basic Medical Science, China Medical University, 77 Puhe Road, Shenyang, Liaoning 110001, P.R. China E-mail: doctorwangdanan@126.com

Key words: nTreg, iTreg, CD8+ Treg, inflammatory bowel disease, dextran sodium sulfate and protecting from immune-related mucosal injury during chronic stage in inflammatory bowel disease.

\section{Introduction}

Inflammatory bowel disease (IBD), often referred to as Crohn's disease or ulcerative colitis (UC), is characterized by gastrointestinal tract inflammation-related disorders $(1,2)$. While Crohn's disease can affect any part of gastrointestinal tract, ulcerative colitis only affects the colon and rectum (3). Although genetic factors or microbiota dysregulation were reported to be the primary factors accounting for the occurrence of IBD in current conceptions $(4,5)$, the detailed pathogenesis of IBD still remains unknown.

Growing evidence has indicated that disruption of intestinal microorganism homeostasis will arouse the immune responses against intracellular bacteria and fungi (6). Even though effector $\mathrm{T}$ cells such as $\mathrm{T}$ helper (h) 17 cells, interleukin (IL)-22 producing T cells as well as the cytokines they produced [IL-17A, IL-22, interferon (IFN)- $\gamma$ fight against the pathogens, they may also cause unwanted tissue destruction, immunopathology and autoimmunity (7-9). Therefore, therapies should focus on restraining T-cell responses in IBD.

Regulatory $\mathrm{T}$ cells, by constitutively expressing IL-2 receptor CD25, serve an essential role in maintaining homeostasis thought constraining the reactivity of effector $\mathrm{T}$ cells as well as blocking pro-inflammatory cytokine production to inhibit the progression of IBD $(10,11)$. Similar to conventional Th cells, T regulatory (reg) cells can be divided into different subsets such as natural Treg cells, induced Tregs and CD8 ${ }^{+}$ Treg cells characterized by different patterns of cytokine and chemokine receptors. Currently, the role of different Treg subsets in modulating inflammatory bowel disease has still not been addressed carefully and systematically.

Therefore, in the present study, the authors sought to determine the percentage of different subsets in well-established dextran sodium sulfate (DSS)-induced colitis mimicking chronic intestinal inflammation in mice at different time points. Efforts were also made to evaluate the change of different Treg subsets at different stage of patients diagnosed with ulcerative colitis compared with enrolled healthy controls. 


\section{Materials and methods}

Mice. A total of 30 5-week-old male mice (C57BL/6; weight, 20-22 g) were purchased from Liaoning Changsheng Biotechnology Co., Ltd. (Benxi, China) and kept under specific pathogen-free conditions with free access to food and water and regular 12-h light/dark cycle in the Experimental Animal Center of China Medical University (Shenyang, China). All the animal experiments were conducted according to the Ethnics of China Medical University (no. KY201511).

Human subjects and ethics. Patients diagnosed of ulcerative colitis and treated at The First Affiliated Hospital of Jinzhou Medical School (Jinzhou, China) from August 2014 to August 2015 were recruited into the study. Age- and sex-matched healthy volunteers were also enrolled as healthy controls. Patients details are shown in Table I. Informed consent was obtained from all the subjects and ethics were approved by The First Affiliated Hospital of Jinzhou Medical School (Jinzhou, China; no. KY201511).

Induction of colitis. 3\% DSS (molecular weight, 4,000Da; Sigma-Aldrich; Merck KGaA, Darmstadt, Germany) were added in drinking water for 7 days to induce acute colitis in mice. Regular drinking water was changed from day 8 . Mice were euthanized at days $0,4,7,10,20$ and 30, respectively. Mesenteric lymph nodes (MLN), peripheral blood and the colon were collected at the same time.

Peripheral blood mononuclear cell (PBMC) isolation. Fresh sodium heparinized blood samples were diluted by PBS, carefully layered on Ficoll density gradient and centrifuged at $1,000 \mathrm{x}$ g for $20 \mathrm{~min}$ in room temperature $\left(25^{\circ} \mathrm{C}\right)$. PBMCs were isolated from the interface and transferred into new tubes, washed with PBS for two times.

Lamina proprialymphocytes (LPL) preparation. Colon tissues were removed of Peyer's patches, washed with PBS, cut into 1-2 mm small pieces and shaken in RPMI 1640 (Sigma-Aldrich; Merck KGaA) supplemented with fetal bovine serum (Sigma-Aldrich; Merck KGaA) and $5 \mathrm{mM}$ EDTA (Sigma-Aldrich; Merck KGaA) at $100 \mathrm{x} \mathrm{g}$ and $25^{\circ} \mathrm{C}$ for $20 \mathrm{~min}$. The supernatants were discarded to remove epithelial cells and intraepithelial lymphocytes. The remained fragments were then incubated with $5 \mathrm{ml} 1 \mathrm{mg} / \mathrm{ml}$ collagenase type IV (Sigma-Aldrich; Merck KGaA), shaken at $100 \mathrm{x}$ g and $25^{\circ} \mathrm{C}$ for between $45 \mathrm{~min}$ to $1 \mathrm{~h}$. The procedure was repeated twice. Supernatant of digested solution were collected, carefully layered on 45/75\% discontinuous Percoll gradients (GE Healthcare Life Sciences, Chalfont, UK) and centrifuged at $800 \mathrm{x}$ g for $20 \mathrm{~min}$ at room temperature. LPLs were harvested, washed with PBS and suspended in RPMI 1640 medium.

Flow cytometry. Human fluorescein isothiocyanate-conjugated anti-CD25, phycoerythrin (PE)-conjugated transforming growth factor (TGF)- $\beta$, peridinin chlorophyll protein complex-conjugated anti-CD4, Alexa Fluor 647 conjugated Foxp3, PE-Cy7-conjugated IL-10, BV421-conjugated anti-CD127 and APC/Cy7-conjugated anti-CXCR5 were purchased from BD Pharmingen (San Diego, CA, USA).
Table I. Basic characteristics of ulcerative colitis patients.

Ulcerative colitis $(n=23)$

No. of patients $(\mathrm{M} / \mathrm{F})$

$11 / 12$

Age (year)

$38.6 \pm 3.4$

C-reactive protein (mg/l)

$12.1 \pm 3.8$

No. in acute stage

16

No. in remittent stage

7

Isolated mononuclear cells were stained with various antibody cocktails at $4^{\circ} \mathrm{C}$ for $30 \mathrm{~min}$, then washed with PBS. For intracellular staining, cells were washed, fixed, permeabilized and stained with intracellular antibody following manufacturer's instructions (BD Biosciences, Franklin Lakes, NJ, USA). Stained cells were detected using FACSCalibur flow cytometry (BD Biosciences). The results were analyzed using FlowJo software (version 9; FlowJo, LLC, Ashland, OR, USA).

ELISA. Serum cytokine levels were analyzed using the following commercially available ELISA kits according to the manufacturer's protocol: MMP-2, Total MMP-2 Quantikine ELISA kit (cat. no. MMP200; Quantikine, R\&D Systems, Inc., Minneapolis, MD, USA); thymic stromal lymphopoietin, Legend Max $^{\mathrm{TM}}$ Human TSLP ELISA kit with Pre-coated Plates (cat. no. 434208; BioLegend, Inc., San Diego, CA, USA); MMP-9, Legend Max ${ }^{\mathrm{TM}}$ Human MMP-9 ELISA kit with Pre-coated Plates (cat. no. 440707; BioLegend, Inc.); IL-17A, Legend Max $^{\mathrm{TM}}$ Human IL-17A ELISA kit with Pre-coated Plates (cat. no. 433918; BioLegend, Inc.); IL-25, Human IL-17E/IL-25 AccuSignal ELISA kit (cat. no. KOA0468; Rockland Immunochemicals, Inc., Limerick, PA, USA); and eotaxin, Human Eotaxin ELISA kit (cat. no. KOA0159; Rockland Immunochemicals, Inc.).

Statistical analysis. Comparisons between two groups were performed using Student's t-test and comparisons among multiple groups were performed using one-way analysis of variance followed by Tukey's post-hoc test. Data are expressed as the mean \pm standard error of the mean. $\mathrm{P}<0.05$ was considered to indicate as statistically significant difference.

\section{Results}

Changes of natural regulatory T-cell subsets in DSS-induced colitis. The best-characterized regulatory $\mathrm{T}$ cell population is the natural regulatory $\mathrm{T}$ cell population. To determine whether the natural regulatory $\mathrm{T}$ cell subset serve a role in alleviating intestinal bowel disease-associated mucosal injury, the authors measured the percentage of Foxp $3^{+} \mathrm{IL}-10^{+}$TGF- $\beta^{+}$natural Tregs at different time points in DSS drinking water-treated mice. The proportion of nTregs in LPL, peripheral blood and MLNs was significantly decreased in the acute phage at days 1 and 4 following DSS water treatment. At 7 days following oral DSS administration, nTregs in LPLs and MLNs were significantly elevated compared with the baseline (Fig. 1A and C). 
A

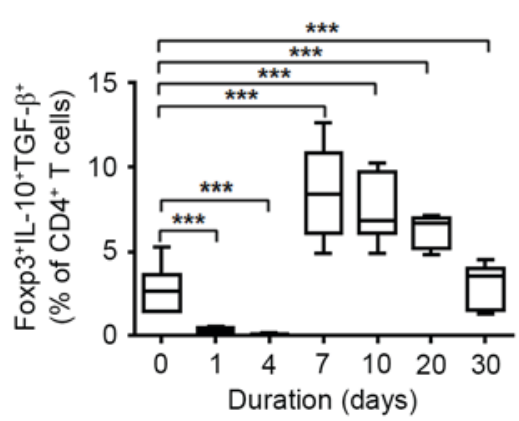

B

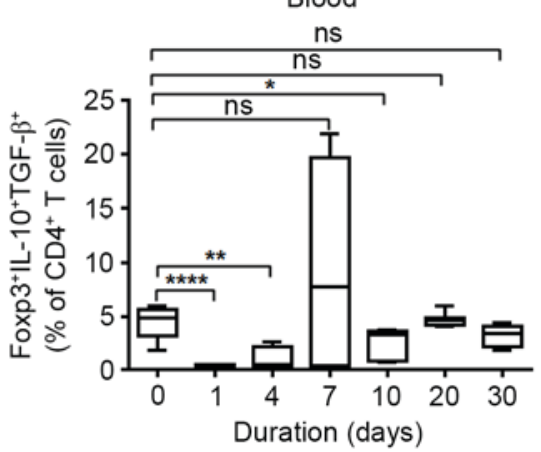

C

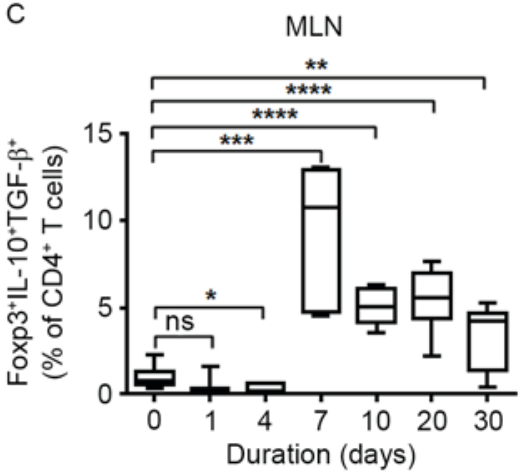

Figure 1. Changes of natural regulatory T-cell subsets in DSS-induced colitis. B6 mice were orally administered with 2.5\% DSS drinking water. Peripheral blood mononuclear cells were isolated from (A) LPLs, (B) peripheral blood and (C) MLNs and analyzed to identify the percentage of Foxp3 $3^{+}$IL- $10^{+}$TGF- $\beta^{+}$ natural regulatory $\mathrm{T}$ cells at different time points. Data are presented as the mean \pm standard error of the mean. ${ }^{*} \mathrm{P}<0.05,{ }^{* * *} \mathrm{P}<0.01,{ }^{* * * *} \mathrm{P}<0.001$ and ${ }^{* * * * *} \mathrm{P}<0.0001$ as indicated. LPLs, lamina propria lymphocytes; MLNs, mesenteric lymph nodes; IL, interleukin, TGF- $\beta$; tumor necrosis factor- $\beta$; DSS, dextran sodium sulfate; ns, not significant.

A

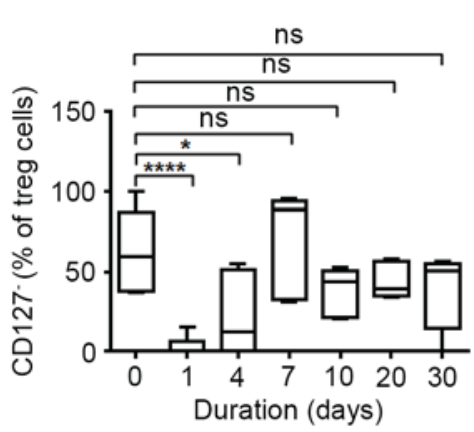

B

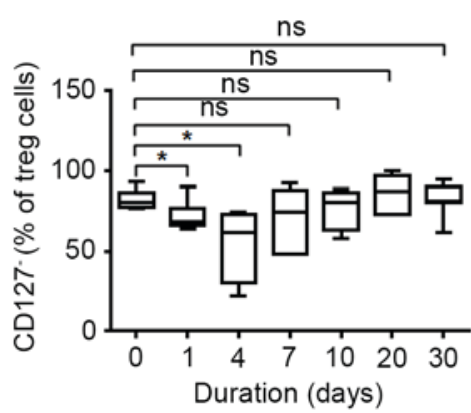

C

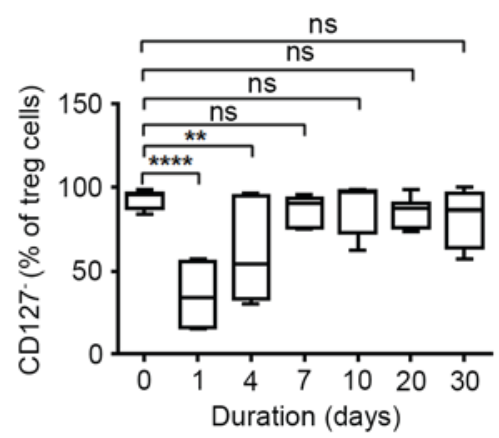

Figure 2. Level of induced regulatory T-cell subsets in DSS-induced colitis. B6 mice were orally administered with 2.5\% DSS drinking water. Peripheral blood mononuclear cells were isolated from (A) LPLs, (B) peripheral blood and (C) MLNs and analyzed to identify the percentage of Foxp3 ${ }^{-}$IL-10 $0^{+}$TGF- $\beta^{-}$induced regulatory $\mathrm{T}$ cells at different time points. Data are presented as the mean \pm standard error of the mean. ${ }^{*} \mathrm{P}<0.05,{ }^{* * *} \mathrm{P}<0.01,{ }^{* * * *} \mathrm{P}<0.001$ and ${ }^{* * * * *} \mathrm{P}<0.0001$ as indicated. LPLs, lamina propria lymphocytes; MLNs, mesenteric lymph nodes; IL, interleukin, TGF- $\beta$; tumor necrosis factor- $\beta$; DSS, dextran sodium sulfate; ns, not significant.

Although slightly elevated nTregs were observed in PBMCs at 7 days, it decreased to a normal level at the chronic stage of DSS-induced IBD (Fig. 1B).

The level of induced regulatory T-cell subsets in DSS-induced colitis. The frequency of Foxp3- IL- $10^{+}$TGF- $\beta^{-}$induced Tregs (Tr1) in LPLs, MLNs and PBMCs was also measured. As presented in Fig. 2, DSS treatment significantly decreased the percentage of $\operatorname{Tr} 1$ cells at days 1 and 4. During the recovery time, the frequency of Tr1 cells continued declining in both LPLs and peripheral blood. Few changes were observed in MLNs at various time points by DSS-treatment.

Analysis of CD127-induced regulatory T-cell frequency in DSS-induced colitis. Following this, the authors determined the change of CD127-inducible (i) Treg population in DSS-induced colitis by flow cytometry. As indicated in Fig. 3, a significantly lower percentage of CD127- Treg was observed in LPLs, MLNs and peripheral blood at days 1 and 4 following DSS treatment. However, the frequency of CD127- Treg cells was only slightly restored but remained at a low level following 7 days in DSS-treated mice.
The proportion of $C D 8^{+}$Tregs in DSS-treated mice. As a small proportion of $\mathrm{CD}^{+}$Tregs were also able to inhibit hyper-responsive effective $\mathrm{T}$ cells in autoimmune diseases, efforts were made to determine the role of $\mathrm{CD}^{+}$Tregs in DSS-induced murine IBD. The proliferation of $\mathrm{CD}^{+}$Tregs was only enhanced in LPL at 7 days following DSS treatment, but reduced to a normal level at days 10, 20 and 30 and no significant differences in MLNs and PBMCs was discovered at any stage compared with the baseline (Fig. 4).

The frequency of Treg subsets in peripheral blood of patients with ulcerative colitis. A total of 23 patients diagnosed of ulcerative colitis were recruited and serum inflammatory cytokines were measured. Higher levels of eotaxin, IL-17A, IL-25 but lower levels of thymic stromal lymphopoietin, matrix metalloproteinase (MMP)-2, MMP-9 were identified compared with healthy controls (Fig. 5). The frequency of four Treg subsets in peripheral blood was also measured by flow cytometry. In good accordance, decreased percentage of nTregs were reported in peripheral blood of patients with UC in the acute stage compared with healthy controls and during remittent stage, a significantly elevated percentage of nTregs 
A

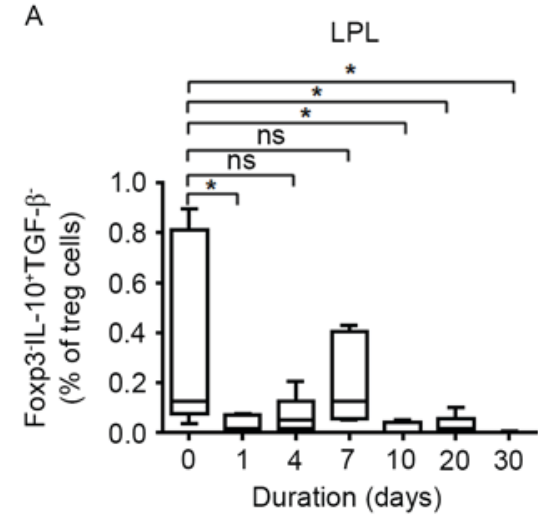

B

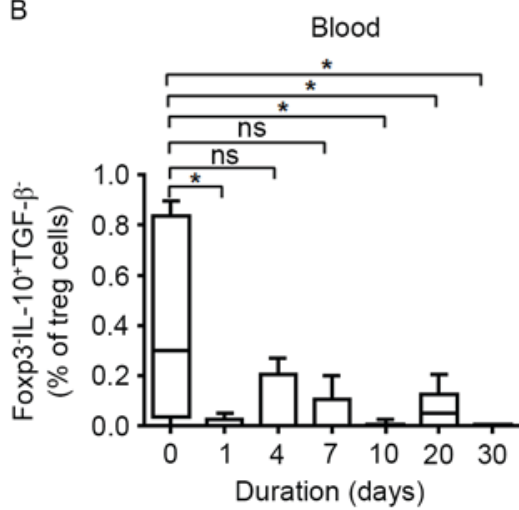

Figure 3. Analysis of CD127 regulatory T-cell frequency in DSS-induced colitis. B6 mice were orally administered with 2.5\% DSS drinking water. Peripheral blood mononuclear cells were isolated from (A) LPLs, (B) peripheral blood and (C) MLNs and analyzed to identify the percentage of CD127 regulatory T cells at different time points. Data are presented as mean \pm standard error of the mean. ${ }^{*} \mathrm{P}<0.05$ as indicated. LPLs, lamina propria lymphocytes; MLNs, mesenteric lymph nodes; DSS, dextran sodium sulfate; ns, not significant.

A

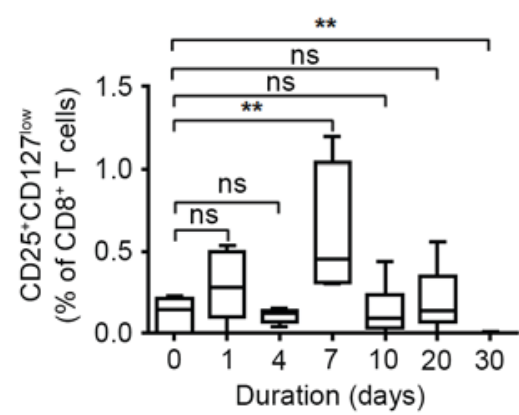

LPL

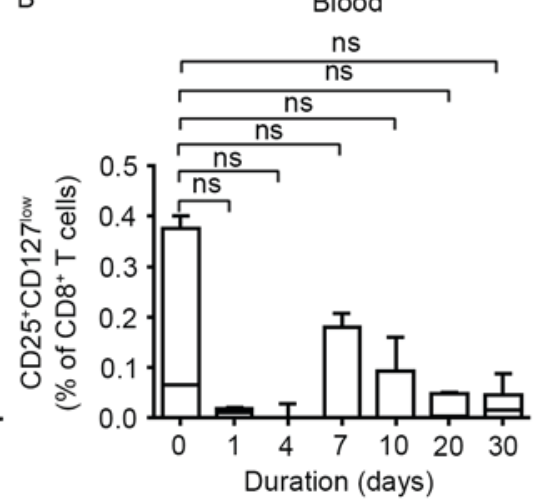

C

B
C

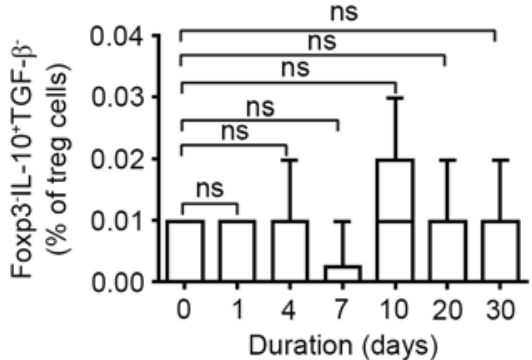

MLN

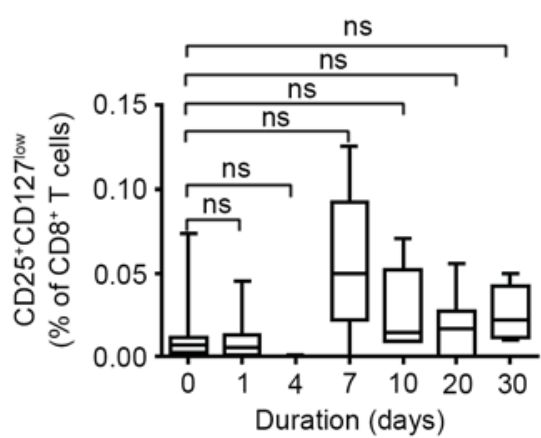

Figure 4. The proportion of CD8 ${ }^{+}$Tregs in DSS-treated mice. B6 mice were orally administered with $2.5 \%$ DSS drinking water. Peripheral blood mononuclear cells were isolated from (A) LPLs, (B) peripheral blood and (C) MLNs and analyzed to identify the percentage of CD8 ${ }^{+}$Tregs at different time points. Data are presented as the mean \pm standard error of the mean. ${ }^{* *} \mathrm{P}<0.01$ as indicated. LPLs, lamina propria lymphocytes; MLNs, mesenteric lymph nodes; Tregs, regulatory T cells; DSS, dextran sodium sulfate; ns, not significant.

was observed (Fig. 6A). The level of $\mathrm{CD}^{+}$Tregs decreased significantly in the acute stage and this was not recovered in remittent stage (Fig. 6D). Both CD127- Tregs and Tr1 did not present any increased tendency either in acute stage and remittent stage (Fig. 6B and C). Collectively, these data in humans and mice suggested that Foxp $3^{+} \mathrm{IL}-10^{+} \mathrm{TGF}-\beta^{+}$natural Tregs may exhibit suppressive activity of against activated immune cells and protect from mucosal injury during chronic stage in DSS-induced intestinal bowel disease.

\section{Discussion}

Regulatory $\mathrm{T}$ cells serve an important role in maintaining intestinal homeostasis and preventing unwanted tissue destructions during the progression of IBD by inhibiting the reactivity of effector immune cells $(12,13)$. In the present study, the authors utilized a well-established DSS-induced murine colitis model and measured the percentage of different Treg subsets at different time points. It was observed that Foxp $3^{+} \mathrm{IL}-10^{+}$ TGF- $\beta^{+}$natural Tregs may exhibit suppressive activity against activated immune cells and protect from mucosal injury during chronic stage in DSS-induced intestinal bowel disease.
nTreg cells, identified by the expression of $\mathrm{CD} 4^{+} \mathrm{CD} 25^{+}$ $\mathrm{FOXP}^{+}$by flow cytometry, were the major component of Treg cells and they control the immune cell expansion either by cell-cell contact or through IL-10 or TGF- $\beta$ secretion $(14,15)$. In an earlier study, by quantifying and characterizing nTreg populations in the LPL and peripheral blood of patients with and without IBD, Lord et al (16) observed a significantly enhanced expression of inhibitory receptors CD39, CTLA-4 and PD-1 in inflamed mucosa compared with controls, suggesting that nTregs are effectively activated in IBD patients. In the current study, the percentage of Foxp $3^{+} \mathrm{IL}-10^{+} \mathrm{TGF}-\beta^{+}$natural Tregs was measured at different time points in murine DSS-induced colitis and different stage of UC patients. It was suggested that, in the acute stage, the proportion of nTregs was decreased, but this recovered and expanded in the remittent stage. Consistent with the findings of Lord et al (16), the data also suggested that dysfunction of nTregs may lead to the development of IBD in the acute stage, however, enhanced nTreg proliferation during the chronic stage may contribute to the suppression of immunopathology in IBD.

iTreg cells that develop from conventional $\mathrm{CD} 4^{+} \mathrm{T}$ cells in the periphery can be classified into CD127- $\left(\mathrm{FOXP}^{+}\right)$and 

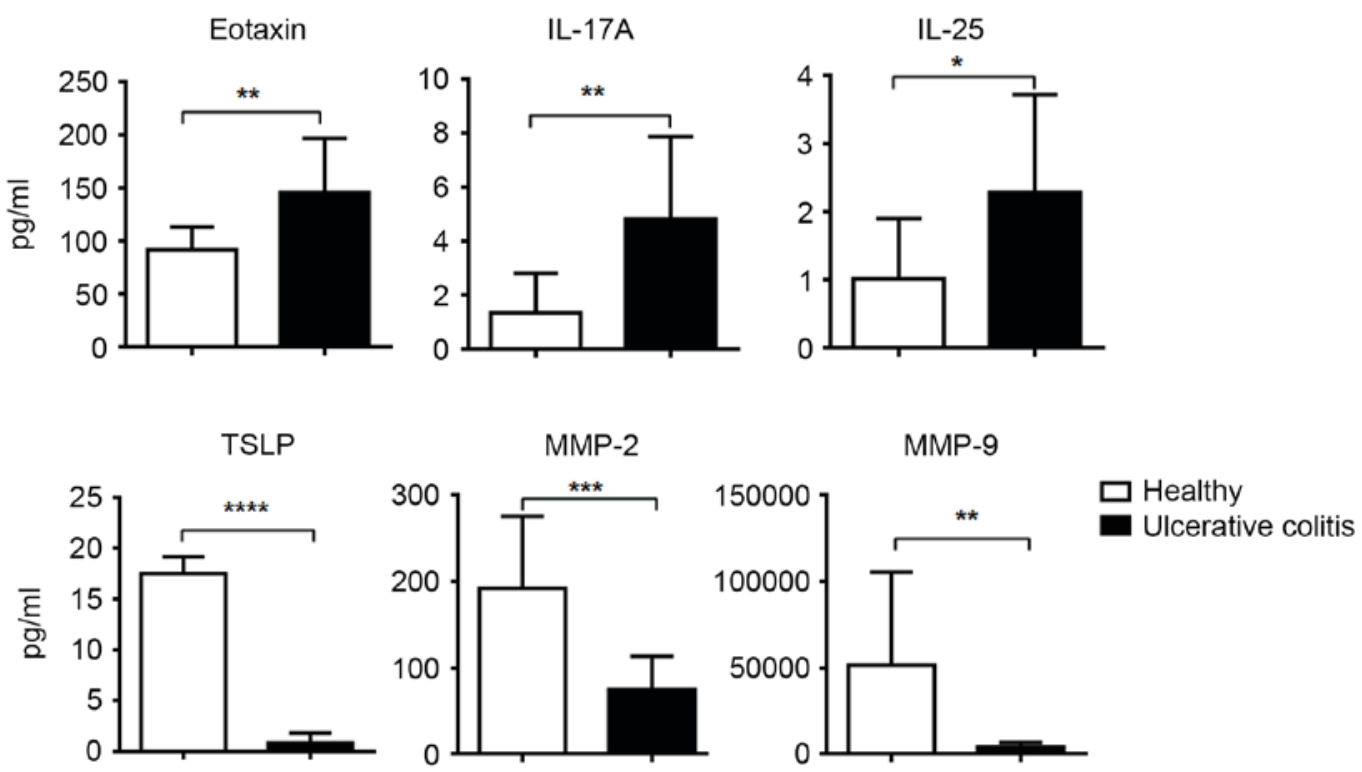

Figure 5. Serum inflammatory cytokines in patients with ulcerative colitis. Serum from ulcerative colitis patients and healthy donors were collected and inflammatory cytokines were determined by ELISA. ${ }^{*} \mathrm{P}<0.05,{ }^{* * *} \mathrm{P}<0.01,{ }^{* * * *} \mathrm{P}<0.001$ and ${ }^{* * * * *} \mathrm{P}<0.0001$ as indicated. IL, interleukin; TSLP, thymic stromal lymphopoietin; MMP, matrix metalloproteinase.

A

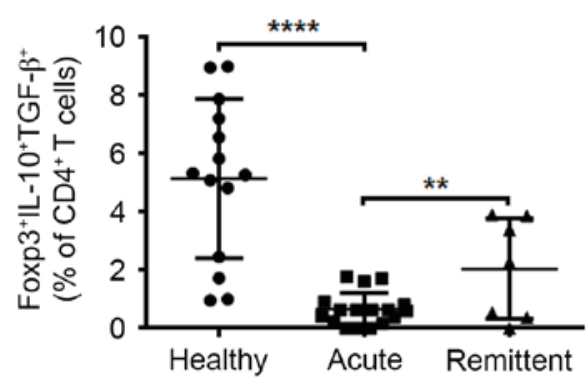

C

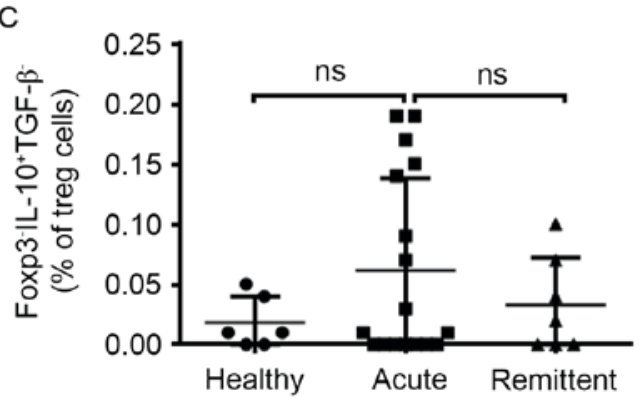

B

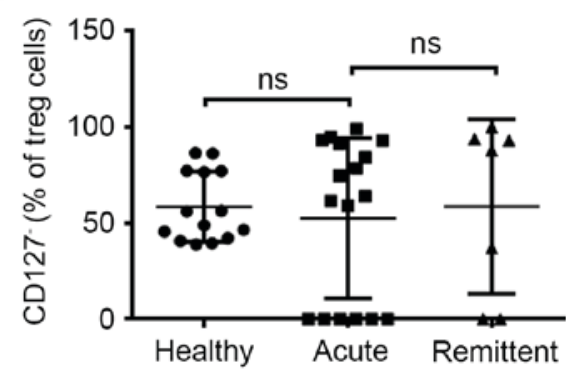

D

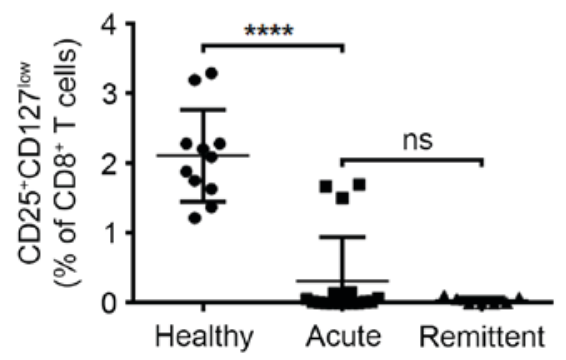

Figure 6. Frequency of Treg subsets in peripheral blood of patients with ulcerative colitis. The percentage of (A) Foxp3 $3^{+}$IL- $10^{+}$TGF- $\beta^{+}$natural Treg cells, (B) CD127- Tregs, (C) Foxp3 $3^{-}$IL-10 ${ }^{+}$TGF- $\beta$-induced Tregs cells and (D) CD8 ${ }^{+}$Tregs in peripheral blood from UC patients and healthy controls are presented. Each dot represents one individual. "* $\mathrm{P}<0.01$ and ${ }^{* * * * *} \mathrm{P}<0.001$ as indicated. Treg, regulator $\mathrm{T}$ cell; IL, interleukin; TGF, transforming growth factor; ns, not significant.

FOXP3-. Both iTreg subsets suppress inflammation through IL-10 production $(17,18)$. Adoptive transfer of iTreg cells can modulate innate and adaptive immune responses, control inflammation and promote epithelial cell homeostasis (19). Furthermore, IL-10 produced by iTreg was sufficient to suppress immune response when is nTreg present but IL-10 is deficient (20). The authors' examinations did not identify any differences in the percentage of Foxp $3^{-} \mathrm{IL}_{-}-10^{+}$TGF- $\beta^{-}$and CD127-iTregs in the acute or remittent stage of human IBD patients compared with healthy individuals. Only a significantly decreased percentage of Foxp $3^{-}$IL- $10^{+}$TGF- $\beta^{-}$and CD127-iTreg was observed in LPL of DSS-induced colitis at 4 and 7 days. Interestingly, the frequency of Foxp3 $3^{-} \mathrm{IL}-10^{+}$ TGF- $\beta^{-}$- iTreg cells became decreased and CD127- iTreg only slightly increased in the chronic stage. The authors suggest that the decreased percentage of Foxp $3^{-}$IL- $10^{+}$TGF- $\beta^{-}$and CD127- iTreg may lead to the autoimmune responses and tissue damage in the acute phage of IBD, however, it could 
not be ruled out that iTreg takes part in promoting intestinal homeostasis during the recovery stage. Further study is required to confirm the role of iTreg cells in IBD.

Since the pioneering work of Rifa'i et al (21) demonstrated $\mathrm{CD}^{+} \mathrm{CD} 122^{+} \mathrm{T}$ subsets that suppress conventional T-cell responses. In addition, $\mathrm{CD} 8^{+}$Tregs are emerging as an important subset of $\mathrm{T}$ suppressors (22). $\mathrm{CD}^{+}$regulatory $\mathrm{T}$-cell populations are also believed to serve an important role in the control of mucosal immunity and Ménager-Marcq et al (23) indicated that naturally occurring $\mathrm{CD}^{+} \mathrm{CD} 28^{-}$regulatory $\mathrm{T}$ lymphocytes can prevent experimental IBD in mice. Analysis the frequency of $\mathrm{CD}^{+}$Treg cells demonstrated different changes in mice and human. While $\mathrm{CD} 8^{+}$Treg cells decrease significantly in patients with acute UC and remained in low levels at the remittent stage, enhanced proliferation of LPL-derived CD8 ${ }^{+}$Treg was observed at 7 days in DSS-induced murine colitis. The authors speculated that $\mathrm{CD}^{+}$Treg served a regulatory role in the late phase of murine IBD and loss of $\mathrm{CD} 8^{+}$Treg activity may contribute to persistent chronic inflammation in human IBD individuals.

In conclusion, the present data highlighted the essential role of Foxp $3^{+} \mathrm{IL}_{-} 10^{+}$TGF- $\beta^{+}$natural Tregs in controlling immune-mediated pathology and consequent tissue damage of IBD in both human and mice. Further studies are still needed to draw a definite conclusion on whether natural Treg therapies were more effective in the cure of human IBD compared to other Treg subsets.

\section{References}

1. Dixon LJ, Kabi A,Nickerson KP and McDonald C: Combinatorial effects of diet and genetics on inflammatory bowel disease pathogenesis. Inflamm Bowel Dis 21: 912-922, 2015.

2. Giusti S, Tani U and Neri E: Inflammatory bowel diseases. In CT Colonography Atlas. Springer: 75-83, 2013.

3. Baumgart DC and Sandborn WJ: Inflammatory bowel disease: Clinical aspects and established and evolving therapies. Lancet 369: 1641-1657, 2007.

4. Jostins L, Ripke S, Weersma RK, Duerr RH, McGovern DP, Hui KY, Lee JC, Schumm LP, Sharma Y, Anderson CA, et al: Host-microbe interactions have shaped the genetic architecture of inflammatory bowel disease. Nature 491: 119-124, 2012.

5. Knights D, Lassen KG and Xavier RJ: Advances in inflammatory bowel disease pathogenesis: Linking host genetics and the microbiome. Gut 62: 1505-1510, 2013.

6. Round JL and Mazmanian SK: The gut microbiota shapes intestinal immune responses during health and disease. Nat Rev Immunol 9: 313-323, 2009.

7. Li L, Shi QG, Lin F, Liang YG, Sun LJ, Mu JS, Wang YG, Su HB, $\mathrm{Xu} \mathrm{B}, \mathrm{Ji} \mathrm{CC}$, et al: Cytokine IL-6 is required in Citrobacter rodentium infection-induced intestinal Th17 responses and promotes IL-22 expression in inflammatory bowel disease. Mol Med Rep 9: 831-836, 2014.
8. Troncone E, Marafini I, Pallone F and Monteleone G: Th17 cytokines in inflammatory bowel diseases: Discerning the good from the bad. Int Rev Immunol 32: 526-533, 2013.

9. Jiang WY, Zhang XF and Zhang HJ: Elevated levels of Th17 cells and Th17-related cytokines in patients with inflammatory bowel disease. J Gastroenterol Hepatol 28: 368-368, 2013.

10. Lim SM, Jeong JJ, Choi HS, Chang HB and Kim DH: Mangiferin corrects the imbalance of Th17/Treg cells in mice with TNBS-induced colitis. Int Immunopharmacol 34: 220-228, 2016.

11. Omenetti S and Pizarro TT: The Treg/Th17 Axis: A dynamic balance regulated by the gut microbiome. Front Immunol 6: 639, 2015.

12. Fantini MC, Rizzo A, Fina D, Caruso R, Becker C, Neurath MF, Macdonald TT, Pallone F and Monteleone G: IL-21 regulates experimental colitis by modulating the balance between Treg and Th17 cells. Eur J Immunol 37: 3155-3163, 2007.

13. Yamada A, Arakaki R, Saito M, Tsunematsu T, Kudo Y and Ishimaru N: Role of regulatory $\mathrm{T}$ cell in the pathogenesis of inflammatory bowel disease. World J Gastroenterol 22: 2195-2205, 2016

14. Chen Y, Sun R, Wu X, Cheng M, Wei $\mathrm{H}$ and Tian Z: CD4+CD25+Regulatory $T$ cells inhibit natural killer cell hepatocytotoxicity of hepatitis $\mathrm{B}$ virus transgenic mice via membrane-bound TGF- $\beta$ and OX40. J Innate Immun 8: 30-42, 2016.

15. Langenhorst D, Gogishvili T, Geyer B and Hunig T: Regulation of IL-10 production in 'natural' regulatory T cells. Wien Klin Wochen 120: 170-170, 2008.

16. Lord JD, Shows DM, Chen J and Thirlby RC: Human blood and mucosal regulatory $\mathrm{T}$ cells express activation markers and inhibitory receptors in inflammatory bowel disease. PLoS One 10: $\mathrm{e} 0136485,2015$.

17. Verhagen J and Wraith DC: Antigen-specific iTreg cells require both IL-10 and CTLA-4 to control autoimmune disease. Immunology 140: 140-140, 2013.

18. Singh A, Seavey C, Horvath K and Mohiuddin M: Induced T regulatory (iTreg) cells suppress $\mathrm{T}$ and $\mathrm{B}$ cell immune responses. Xenotransplantation 18: 271-272, 2011.

19. Haribhai D, Chatila TA and Williams CB: Immunotherapy with iTreg and nTreg cells in a murine model of inflammatory bowel disease. Methods Mol Biol 1422: 197-211, 2016.

20. Schmitt EG, Haribhai D, Williams JB, Aggarwal P, Jia S, Charbonnier LM, Yan K, Lorier R, Turner A, Ziegelbauer J, et al: IL-10 produced by induced regulatory $\mathrm{T}$ cells (iTregs) controls colitis and pathogenic Ex-iTregs during immunotherapy. J Immunol 189: 5638-5648, 2012.

21. Rifa'i M, Kawamoto Y, Nakashima I and Suzuki H: Essential roles of $\mathrm{CD} 8+\mathrm{CD} 122+$ regulatory $\mathrm{T}$ cells in the maintenance of T cell homeostasis. J Exp Med 200: 1123-1134, 2004.

22. Jebbawi F, Fayyad-Kazan H, Merimi M, Lewalle P, Verougstraete JC, Leo O, Romero P, Burny A, Badran B, Martiat P and Rouas R: A microRNA profile of human CD8(+) regulatory $\mathrm{T}$ cells and characterization of the effects of microRNAs on Treg cell-associated genes. J Transl Med 12: 218, 2014.

23. Ménager-Marcq I, Pomié C, Romagnoli P and van Meerwijk JP: CD8+CD28- regulatory $\mathrm{T}$ lymphocytes prevent experimental inflammatory bowel disease in mice. Gastroenterology 131: 1775-1785, 2006. 